\title{
INFORME SOBRE LOS RESTOS DE DALCA DEL MUSEO ETNOGRÁFICO DE ACHAO, CHILOÉ
}

\author{
NICOLÁS LIRA", VALENTINA FIGUEROA" \& ROMINA BRAICOVICH ${ }^{* * * *}$
}

\begin{abstract}
RESUMEN
El presente informe expone el trabajo de documentación y análisis de dos fragmentos de dalca depositados y exhibidos en el Museo Etnográfico de Achao, isla de Chiloé y sus resultados. Este estudio es una aproximación preliminar a estas piezas, que deberá ser profundizado en el futuro con más detalle, tiempo y recursos.
\end{abstract}

PALABRAS CLAVE: dalca, Chiloé, coigüe, navegación, tecnología.

\section{REPORT OF THE PIECES OF DALCA FROM THE ETHNOGRAPHIC MUSEUM OF ACHAO, CHILOÉ}

\footnotetext{
ABSTRACT

This report presents the record and analisys of two pieces of dalca exhibited in the Ethnographic Museum of Achao, Chiloé Island, and its results. This study is a preliminar aproach to these pieces, which in the future should be continued in deeper details with more time and resources.

KEY WORDS: dalca, Chiloé, coigüe, navigation, technology.

Las piezas estudiadas son dos pequeños fragmentos de madera, que se encuentran en exhibición en la primera sala del Museo Etnográfico de Achao, isla de Chiloé. Según la información que se encuentra en vitrina, éstas habrian sido encontradas en la playa Conchas Blancas, en las cercanías de Achao y donadas por don Galvarino Barrientos en la

década de 1960. El trabajo se desarrolló en las dependencias del museo.

\section{EVIDENCIAS MATERIALES DE LA DALCA}

El presente estudio no pretende convertirse en una investigación acabada acerca de la dalca, ni en una recopilación exhaustiva de la información

* Université de Paris 1 Panthéon-Sorbonne; UMR 8096 ARCHAM, 3 rue Michelet, 75006, Francia. nicoliras@yahoo.com.

* Universidad Católica del Norte, Instituto de Investigaciones Arqueológicas y Museo R.P. Gustavo Le Paige (IIAM), Calle Gustavo Le Paige, 380, San Pedro de Atacama, Chile. vfigueroa@ucn.cl.

** Administración de Parques Nacionales Delegación Regional Patagonia, Vice Almirante O'Connor 1188, San Carlos de Bariloche, Río Negro, Argentina. raecovich@yahoo.com.
} 
que se encuentra disponible sobre estas embarcaciones. Varios autores ya se han avocado a esta tarea, y entre los más destacados podemos nombrar a Cooper (1917), Latcham (1930), Lothrop (1932, Fig. 1), Finterbusch (1934) y Medina (1984). Baste decir que la dalca fue la embarcación ampliamente utilizada en el archipiélago de Chiloé. Está compuesta por tres a cinco tablas cosidas entre sí por medio de costuras vegetales y calafateadas con una preparación o betún especial para impermeabilizarlas. Estas embarcaciones se utilizaban tanto para transportarse como para la explotación de los diversos recursos. Presentaban la posibilidad de ser desarmadas por sus costuras y, de esta forma, transportadas fácilmente por tierra a través de los llamados pasos de canoas o "pasos de indios", que por la intrincada geografía de la zona les ahorraban tiempo y energía.

La abundante documentación escrita sobre las dalcas contrasta con la escasez de evidencias materiales de estas embarcaciones, las que se reducen a algunos fragmentos descontextualizados, reproducciones presentes en museos nacionales, $y$ a limitados ejemplares etnográficos completos en el extranjero, todos muy pobremente documentados. Ningún etnógrafo ha registrado el proceso de fabricación de una dalca, y las representaciones iconográficas de ella resultan muy escasas (Carabias et al. 2007). Además, a pesar de que se presume prehispánica, ya que a la llegada de los conquistadores su uso se encontraba extendido en la zona de Chiloé, no hay evidencias de estas antes de 1567. En este sentido, según los antecedentes recopilados, los restos de dalcas conocidos se limitarían a:

1.- Un fragmento de las dalcas descubiertas en 1856 en el lago Nahuelhuapi por Francisco Fonck (1900, p. 186-287) es reproducido por Fintersbuch (1934, p. 26), y de acuerdo a Medina (1984, p. 131) podría encontrarse en los depósitos del Museo de Historia Natural. Hasta el momento no hemos podido localizarlo.

2.- Un modelo de dalca proveniente de Chiloé habría sido incorporado al Museo Nacional de Chile previo a 1880 (Latcham,

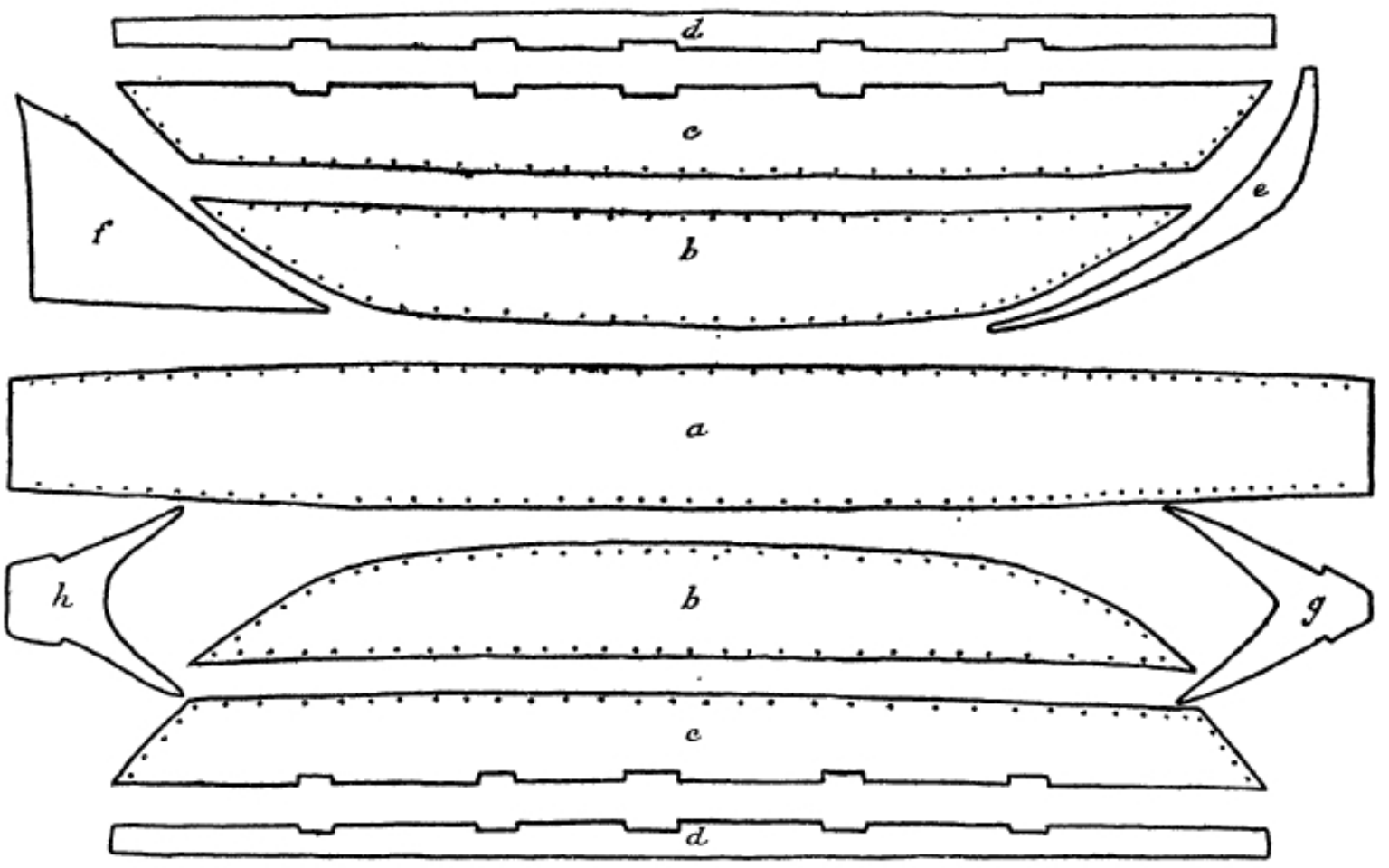

Fig. 1. Ilustración de la reproducción de una dalca del Museo de Historia Natural de Santiago y su forma de ensamblaje según Lothrop (1932). 

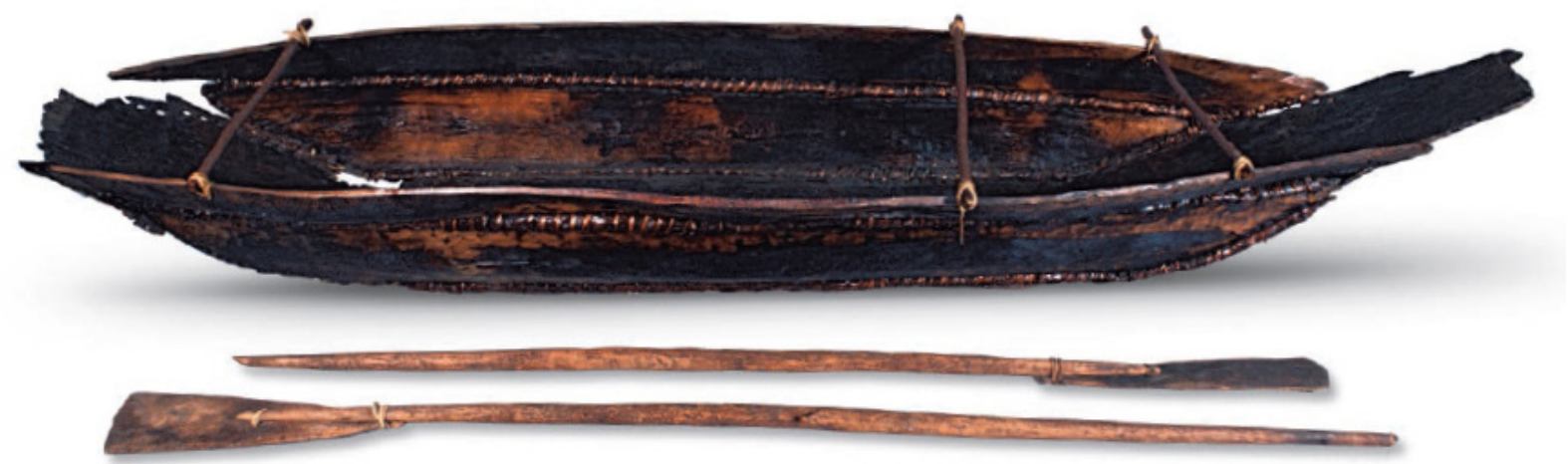

Fig. 2. Dalca del museo etnográfico de Estocolmo. Fue llevada a Suecia en 1907 por el botánico y naturalista Carl Skottsberg luego de una misión científica en la Patagonia. Es el único ejemplar conocido completo que se encuentra ensamblado. Colección Etnografiska museet, Estocolmo (Lira \& Legoupil, 2014).

1930, p. 9-10). Hasta el momento tampoco hemos podido localizarlo.

3.- Restos de otra dalca habrían sido recuperados en Chiloé en 1935 y observados por Junius Bird (1938, p. 254). Tampoco se tiene mayores antecedentes sobre estos fragmentos.

4.- Un fragmento de dalca que se encuentra en el Museo de Historia Natural de Santiago.

5.- En el extranjero, se conservan ejemplares de dalcas etnográficas recolectadas en Patagonia austral por Carl Skottsberg hacia 1910, en el Världskultur museet de Gotemburgo y el Etnografiska Museet de Estocolmo, Suecia (Fig. 2). Tanto estas como la anterior forman parte de una investigación mayor que se encuentra en su fase de redacción (Lira, 2015).

6.- Una excepción a este escenario lo constituyen los restos de dalca recuperados del lago Chapo en 1999, los que actualmente forman parte de la colección del Museo Juan Pablo II de Puerto Montt. Considerando la escasez de evidencias directas de este tipo de transportes marítimos y su potencial valor científico patrimonial, en 2005 un equipo de arqueólogos desarrolló una evaluación y diagnóstico técnico de estas piezas con miras a su futura exhibición y puesta en valor (Lira, 2006; Carabias et al. 2007).

Es precisamente este contexto de 'escasez de evidencias materiales' el que torna altamente valorable los fragmentos de dalca de Achao, ameritando, a nuestro entender, una mayor profundización de su estudio en el futuro.

\section{REGISTRO DE LOS FRAGMENTOS DE DALCA DE ACHAO}

\section{Fragmento 1 (DACH1-F1)}

Tabla 1. Dimensiones Fragmento 1.

\begin{tabular}{cc}
\hline Dimensiones & \\
\hline Largo máximo & $103 \mathrm{~cm}$ \\
Ancho máximo & $22 \mathrm{~cm}$ \\
Ancho mínimo & $6 \mathrm{~cm}$ \\
Espesor máximo & $3,45 \mathrm{~cm}$ \\
Espesor mínimo & $2,08 \mathrm{~cm}$ \\
\hline
\end{tabular}

Este fragmento presenta una sola hilera de agujeros en lo que denominamos borde inferior (Fig. 3). Denominamos a este borde como inferior ya que pareciera proyectar una curvatura que se correspondería con la que presentan los tablones de las bordas en su parte más baja (Fig. 1). El extremo donde se encuentran los agujeros sería, al parecer, el único completo, es decir que concluye donde originalmente terminaba. El resto de este tablón de dalca no se conservó en su estado original, por lo que no se puede saber si habría agujeros en el extremo opuesto, o en el costado, ni tampoco cual habría sido la dimensión real de la tabla.

Se denominó a la superficie convexa del fragmento como cara exterior (Fig. 4) y a la superficie cóncava como cara interior (Fig. 5). Presenta huellas de exposición intencional 
Borde Superior

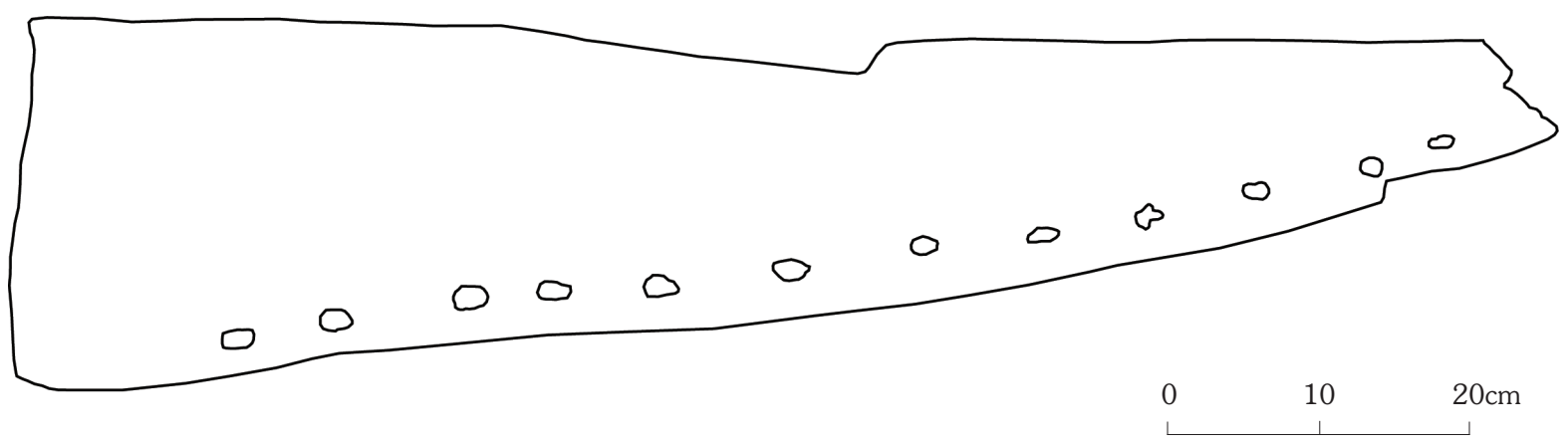

Fig. 3. Dibujo a escala del Fragmento 1 en el que se observa el borde superior y los agujeros en el borde inferior.

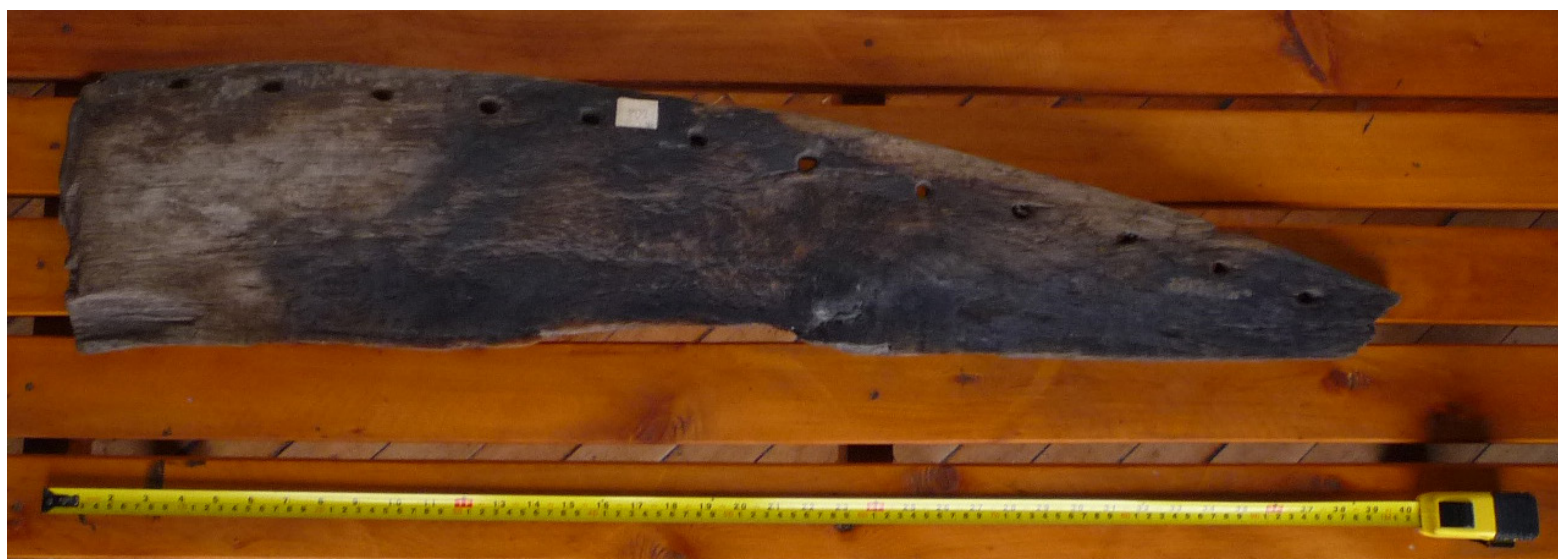

Fig. 4. Cara exterior Fragmento 1. (Escala cinta métrica 1m).

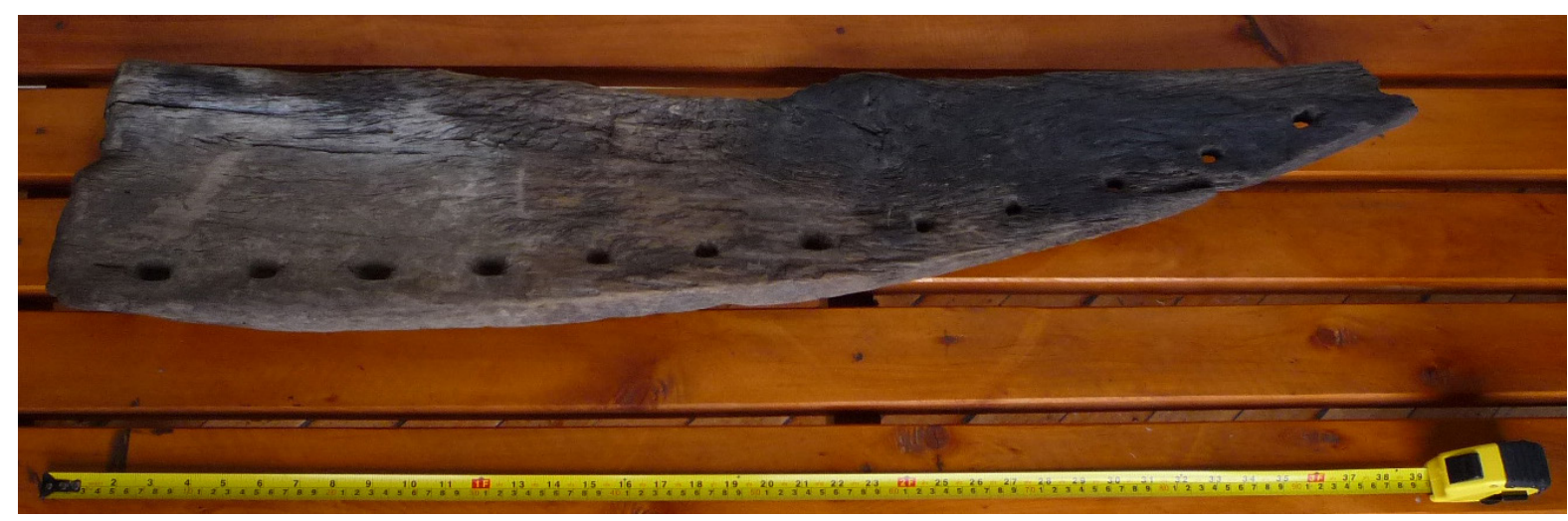

Fig. 5. Cara interior Fragmento 1.

y controlada al fuego en ambas caras, lo que resulta en que la superficie se encuentra bastante carbonizada, y que la pieza presente una ligera curvatura (Fig. 6), la que también habría sido intencionalmente realizada durante este proceso.

\section{Agujeros}

Los agujeros, por donde habrían pasado las fibras vegetales con que se unían o cosían los tablones entre sí, se ubican en el borde inferior, a 

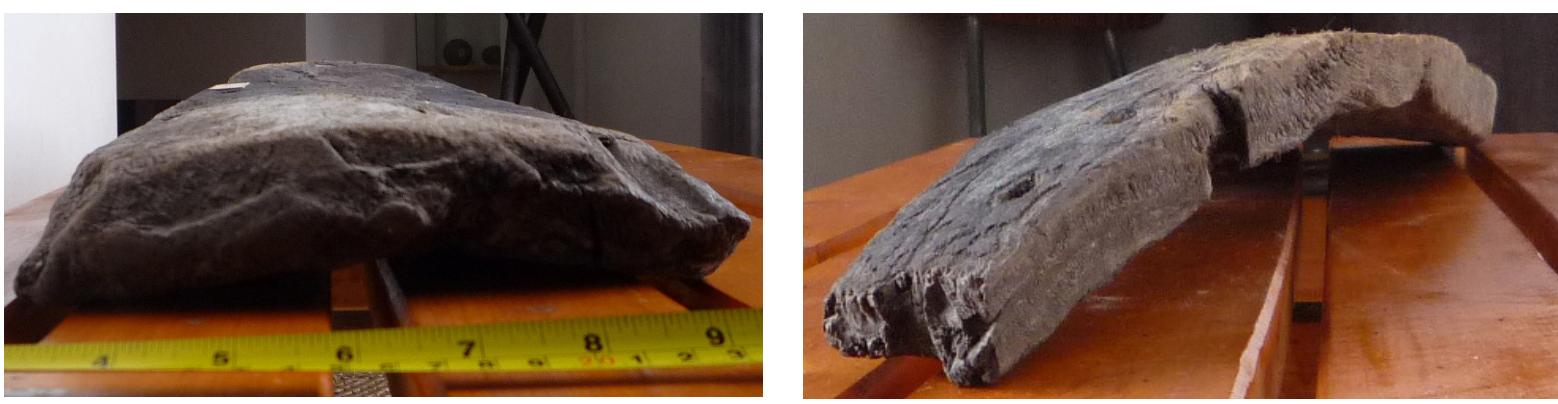

Fig. 6. Vista de la curvatura del Fragmento 1, cara exterior.

lo largo de todo el fragmento. Esta zona presenta un mayor espesor que el resto de la pieza, mientras que el espesor mínimo se registra en el extremo opuesto.

Se alcanzan a apreciar 12 agujeros en total.
En algunos de ellos se observa fibra vegetal, de la que se tomó una muestra (Fig. 7). Los agujeros son irregulares, presentando una forma cuadrangular en su exterior y redondeada en el interior (forma cuadrangular y perfil cónico). No presentan marcas
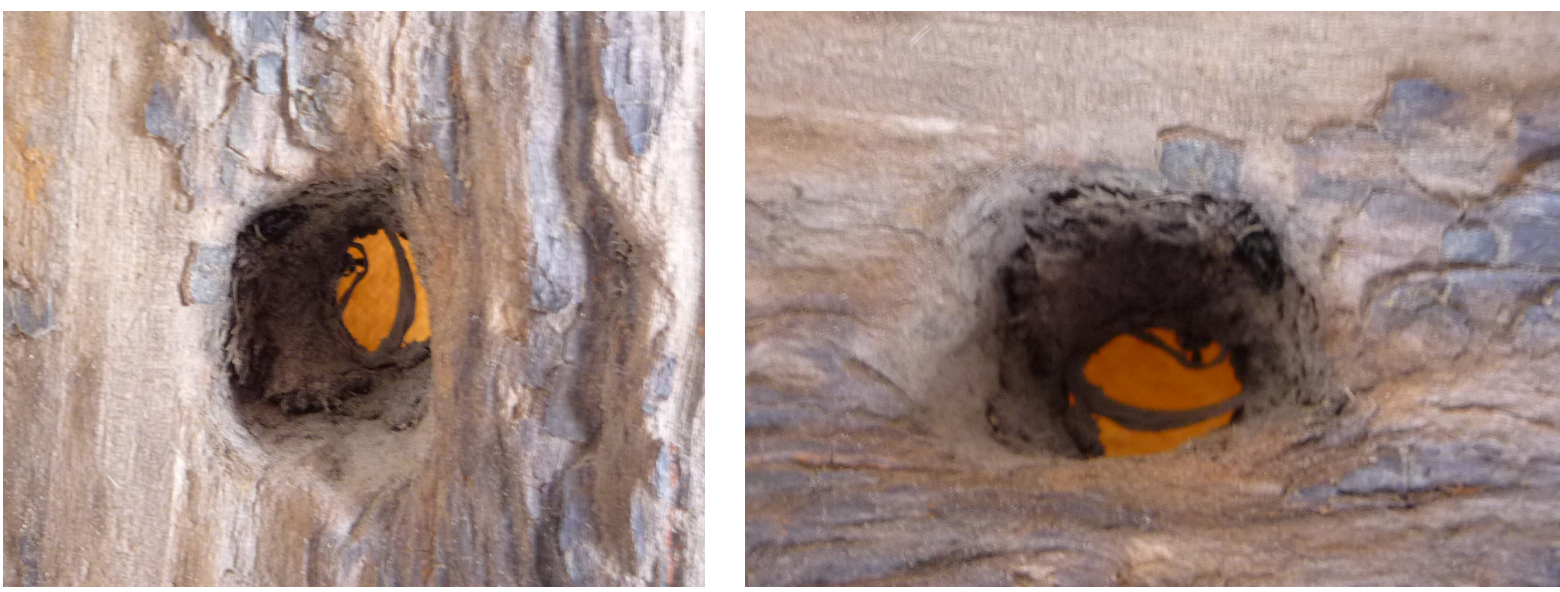

Fig. 7. Agujero con fibras vegetales en su interior, tomado desde dos vistas diferentes.
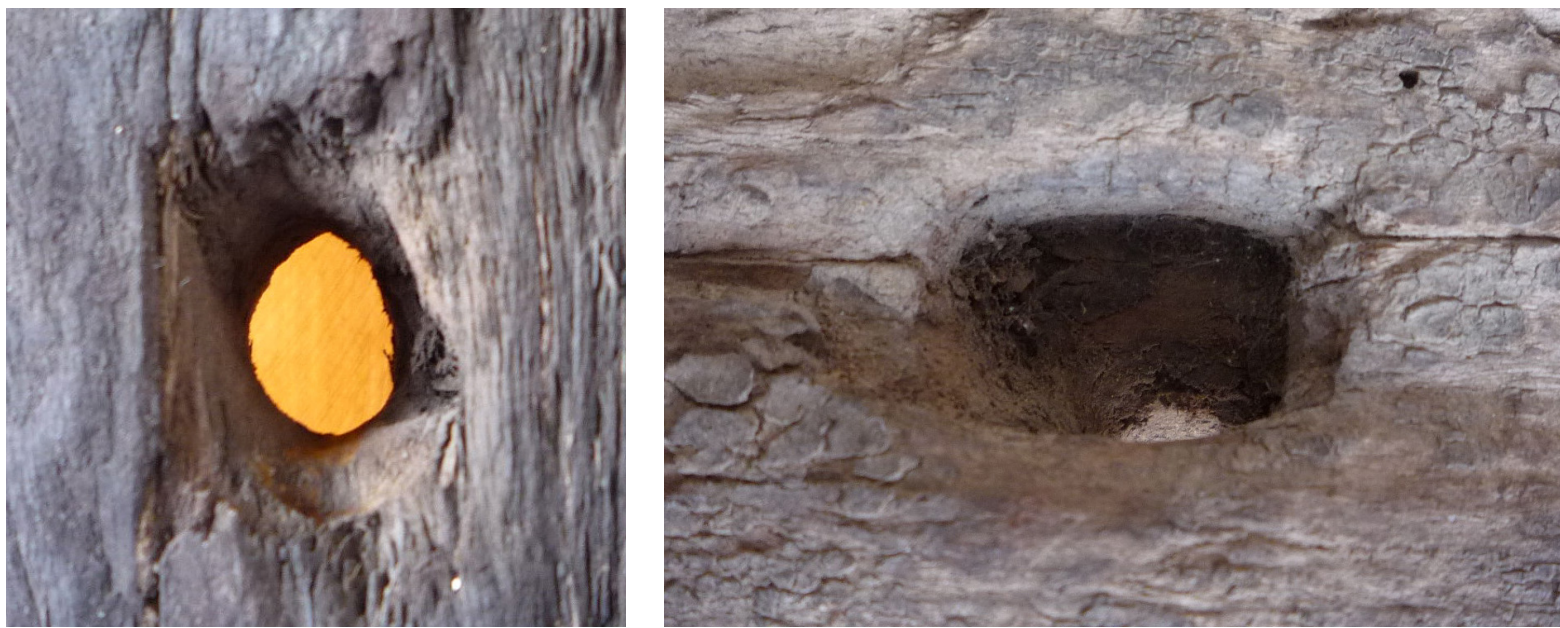

Fig. 8. Vista de dos agujeros distintos del Fragmento 1. Se aprecia la forma cuadrangular en el exterior y redondeada en el interior de los agujeros. 
de haber sido expuestos al fuego o carbonización en su interior, aunque varios de ellos si las presentan a su alrededor. Esto muestra que los agujeros habrían sido realizados después de que la pieza fue sometida a la exposición al fuego, y de que no se habría hecho uso de éste durante su proceso de manufactura (Fig. 8).

En cuanto a sus medidas, estas fueron tomadas por ambas caras, interior y exterior, ya que no eran simétricos. Sus valores varían entre 1,86-1,1 cm de alto y 2,7-1,32 cm de ancho en su cara interior; y $1,36-0,98 \mathrm{~cm}$ de alto y 1,68-1,16 $\mathrm{cm}$ de ancho en su cara exterior. Los agujeros son en casi todos los casos de mayor tamaño por la cara interior, por lo que se puede plantear que posiblemente éstos habrían sido tallados o perforados desde la cara interior y traspasaron hacia la cara exterior. Sólo en tres de ellos (agujeros 9, 10 y 11) las dimensiones del ancho fueron superiores en la cara exterior que en la interior, mientras que las dimensiones de su altura se mantuvieron (agujero 9) o redujeron (agujeros 10 y 11) en la cara exterior respecto de la cara interior. Esto podría deberse ya sea a un acabado adicional de estos agujeros por la cara exterior, posiblemente debido a alguna dureza especial de la madera en esa zona, como también a un desgaste de estos agujeros provocado por las costuras de fibra vegetal. Junto con esto, en la mayoría de los casos los agujeros presentan una forma más cuadrangular en la cara interior que en la cara exterior, como si se hubiera utilizado una herramienta similar a un formón para su tallado. Probablemente una herramienta de ese tipo haya sido utilizada para iniciar el proceso de desbaste y luego se haya regularizado con otro tipo de herramientas. Estos no son rectos, sino que se inclinan hacia el extremo superior.

\section{Fragmento 2 (DACH1F2)}

Tabla 2. Dimensiones Fragmento 2.

\begin{tabular}{cc}
\hline Dimensiones & \\
\hline Largo máximo & $96 \mathrm{~cm}$ \\
Ancho máximo & $29,5 \mathrm{~cm}$ \\
Espesores & $1,48 \mathrm{~cm} / 2,17 \mathrm{~cm} / 1,94 \mathrm{~cm}$ \\
\hline
\end{tabular}

Observaciones: Tiene 2 extremos con agujeros. Es mucho más delgado que el Fragmento 1.

Este fragmento presenta dos extremos con agujeros (los del borde inferior están rotos o incompletos) lo que podría corresponder al ángulo del borde o final de un tablón de la borda de la dalca. Sugerimos esta interpretación al observar que la única zona donde pueden encontrarse agujeros en ambos bordes de un tablón de dalca es justamente en los extremos de los tablones de las bordas (Fig. 1). El que posee más agujeros fue llamado borde superior, sólo a manera de referencia (Fig. 9). Al igual que en el fragmento 1, se denominó a la superficie convexa del fragmento como cara exterior (Fig. 10) y a la superficie cóncava como cara interior (Fig. 11). La parte apuntada o angulosa del lado izquierdo de este fragmento presenta algunas dificultades para su interpretación, ya que parecería estar tallada intencionalmente en esa forma. Esto plantea algunas dudas acerca si se trataría de un fragmento

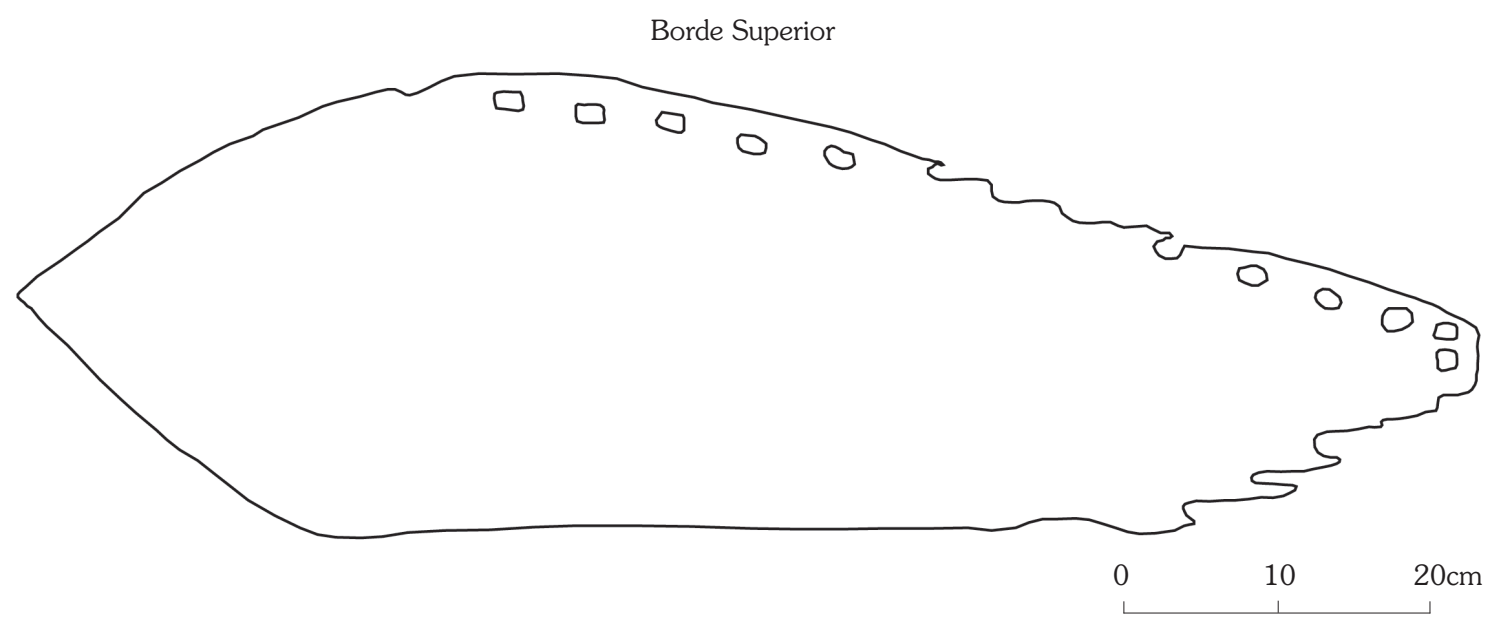

Fig. 9. Dibujo a escala del Fragmento 2, en el que se aprecia el borde superior y los agujeros superiores e inferiores. 
de un tablón de mucho mayor tamaño, el que se habría fracturado y al cual la erosión ha regularizado en esta forma; o si se trata de una pequeña pieza tallada de esta manera. Sin embargo, se requieren observaciones más detenidas para realizar esta última afirmación y por el momento nos inclinamos más por la primera. En el costado derecho presenta dos agujeros (uno de los cuales está quebrado) en posición vertical, y luego en la parte inferior se aprecian agujeros rotos en dirección diagonal a los del borde superior. Esto nos estaría indicando que podría tratarse del extremo de una tabla de dalca de una de las bordas, a partir de las observaciones que hemos realizado de otros ejemplares. Sin embargo, nuevamente, es necesario realizar observaciones más detalladas para confirmar este dato. La zona inferior que no presenta agujeros está bastante erosionada y desgastada, como si hubiera sido tallada, pero creemos que sería resultado de la erosión natural. Se observa una exposición diferencial a la erosión, estando más expuesto el extremo inferior, ya que sus agujeros se encuentran incompletos.

\section{Agujeros}

En los agujeros se apreciaron algunas huellas de manufactura y uso (Figs. 12 y 13). Como en los agujeros del Fragmento 1, posiblemente estos podrían haber sido realizados siguiendo un diseño cuadrangular, siendo igualmente desbastados con una herramienta similar a un formón. También se encuentran algunas huellas de tipo circular, y varios agujeros se encuentran más redondeados, aunque no está claro si ambos elementos son producto del desgaste y uso o del proceso de manufactura con una herramienta diferente.

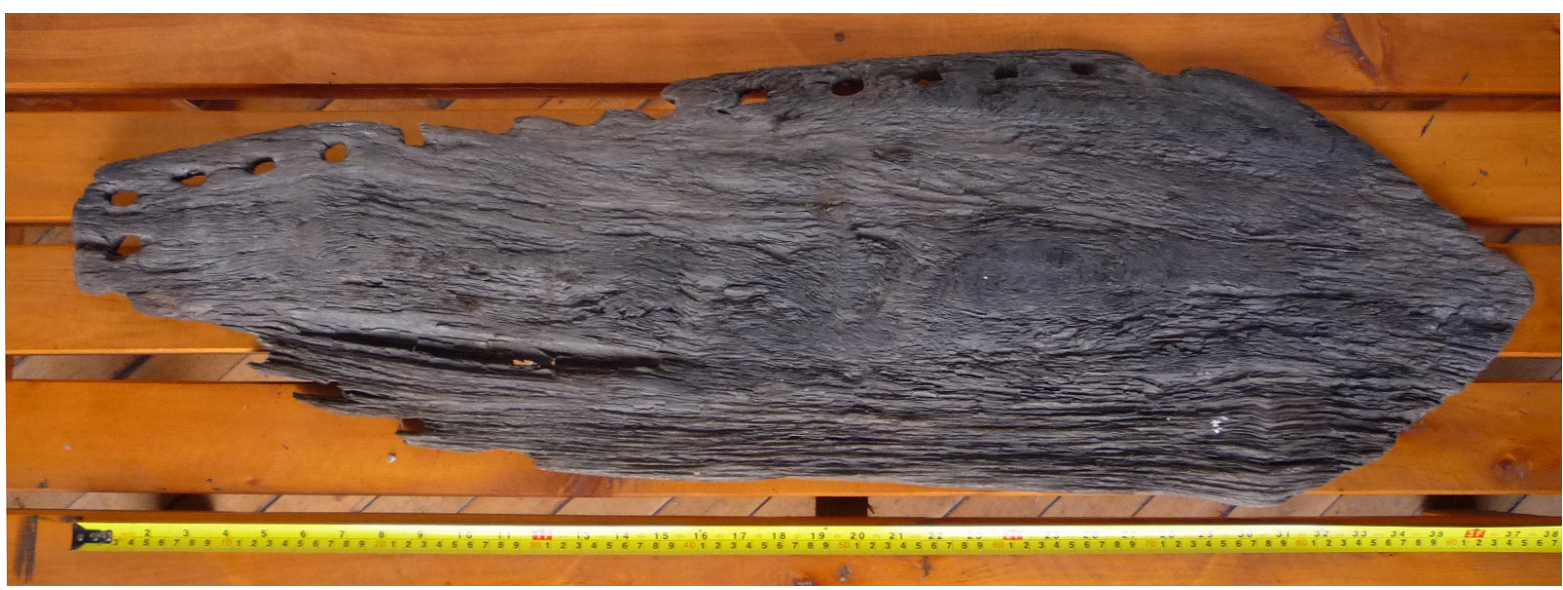

Fig. 10. Cara exterior Fragmento 2. (Escala cinta métrica 1m).

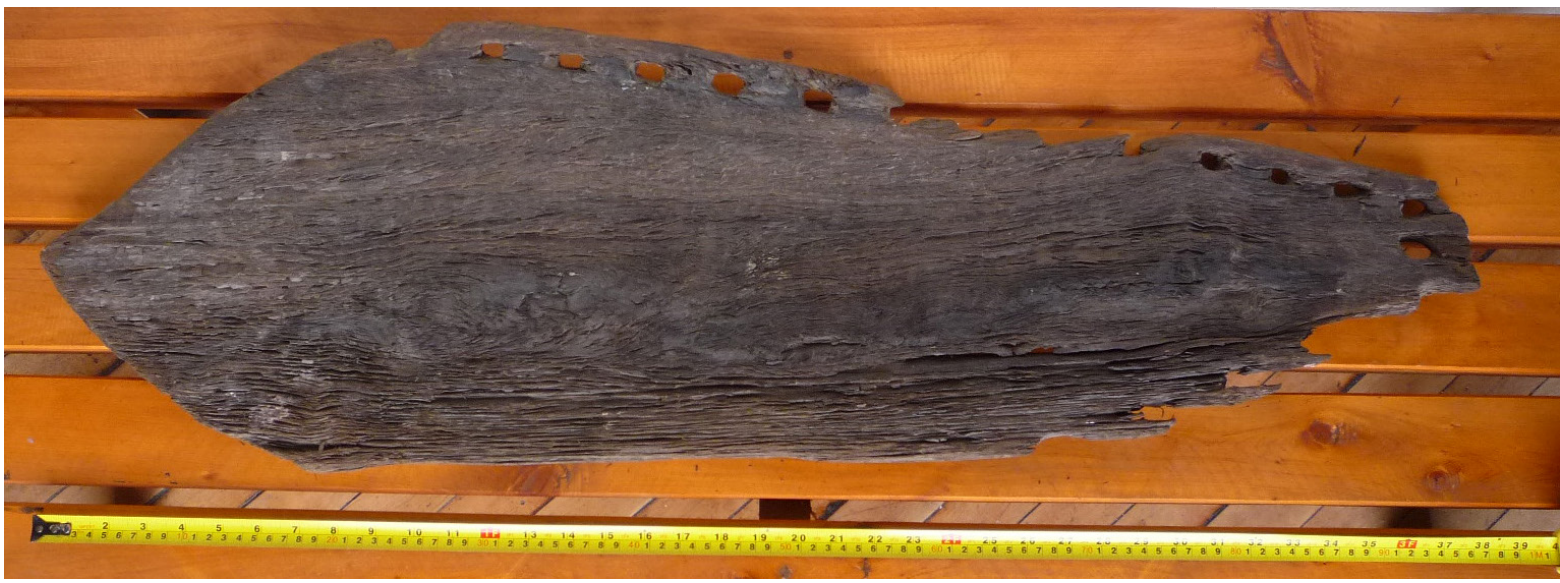

Fig. 11. Cara interior Fragmento 2. 

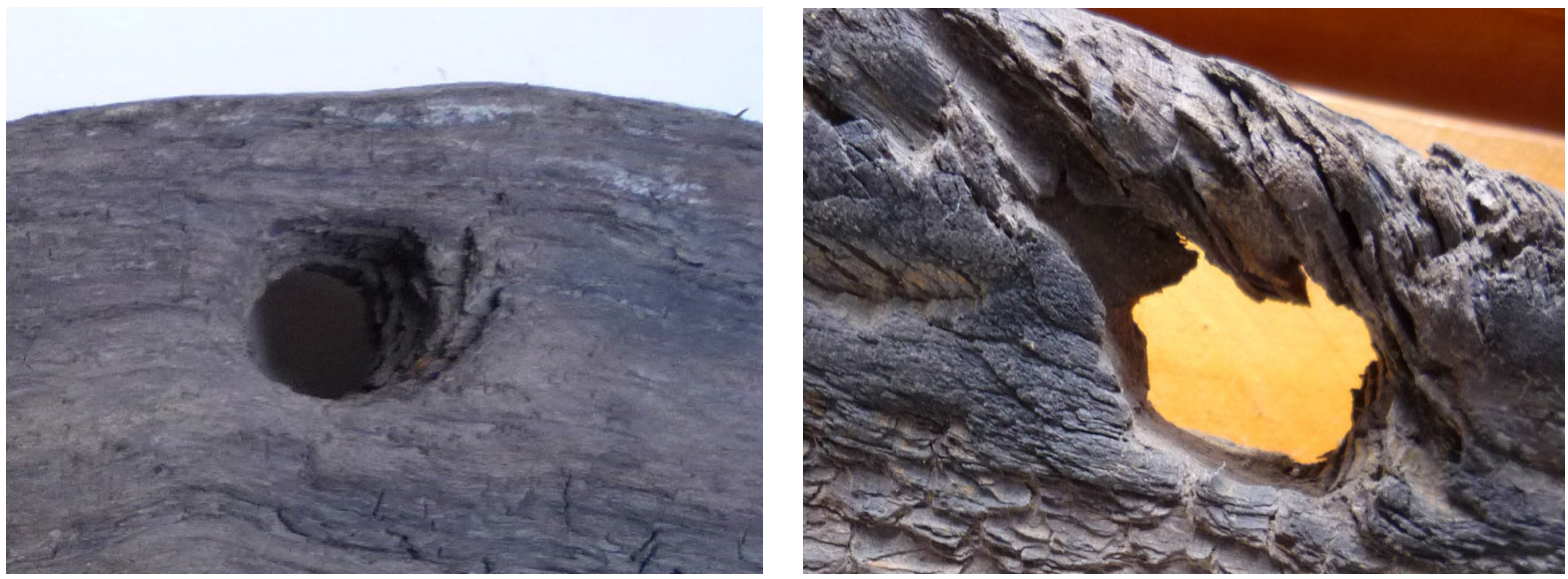

Fig. 12. Detalles de huellas de manufactura y uso de agujeros del Fragmento 2.
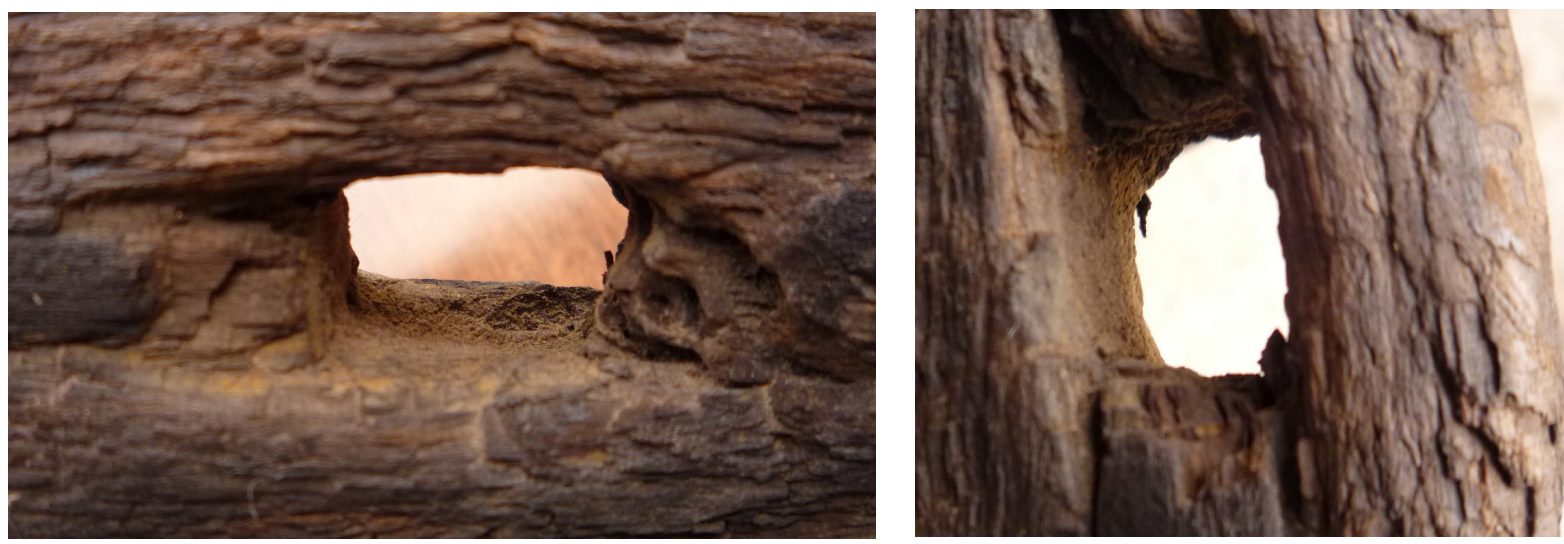

Fig. 13. Detalle de huellas de manufactura de agujeros del Fragmento 2. Se aprecia su forma cuadrangular y parte del desbaste realizado con una herramienta similar a un formón.

En cuanto a las dimensiones, solo se tomaron aquellas que pertenecían a agujeros completos. Se comenzó en la parte superior extremo izquierdo con el agujero 0, del cual sólo se adivina levemente la forma, y luego en sentido del reloj hasta los agujeros 13 y 14 que son los dos del extremo derecho. Todos los agujeros del borde inferior estaban incompletos, por lo que no se pudo obtener sus dimensiones. En total se contaron 20 agujeros, obteniéndose las dimensiones sólo en 10 de ellos. Nuevamente se tomaron medidas en ambas caras del fragmento. Sus dimensiones varían entre 1,96-1,16 cm de alto y $2,35-1,38 \mathrm{~cm}$ de ancho en su cara interior; y $1,62-1,03 \mathrm{~cm}$ de alto y 2,22-1,18 cm de ancho en su cara exterior. Al igual que en el fragmento 1 , los agujeros son en general de mayor tamaño por la cara interior, lo que permite sugerir que estos habrian sido perforados desde esta cara hacia la cara exterior.
Sólo los agujeros 1 y 11 son levemente más anchos por su cara exterior; mientras que los agujeros 10, 11 y 13 son levemente más altos por su cara exterior, lo que puede explicarse de la misma manera que en el fragmento 1, por un acabado adicional desde la cara exterior (producto de una mayor dureza de la madera en esa zona) o por desgaste provocado por las costuras de fibra vegetal.

\section{ANÁLISIS DE LABORATORIO}

Durante el registro y observación de las piezas se tomaron pequeñas muestras de madera para su análisis en laboratorio. El objetivo era identificar la especie de la madera con que se realizaron estas piezas de dalca, y fechar al menos uno de ellos mediante el método de radiocarbono 14 . 


\section{Identificación de especie/taxa}

La identificación de la especie de la madera de estos fragmentos de dalca se realizó a través del análisis microscópico de la anatomía de la madera y se apoyó en el empleo de claves y comparación con muestras de la xiloteca del Instituto de Tecnología de Productos Forestales, Facultad de Ciencias Forestales de la Universidad Austral de Chile. Este análisis fue realizado por el doctor Juan Eduardo Díaz-Vaz, anatomista de maderas y académico de dicha universidad, asistido en el proceso por Nicolás Lira, arqueólogo y uno de los autores de este estudio.

La observación microscópica de ambos fragmentos arrojó similares resultados, siendo las principales características: Poros solitarios y que en algunos casos se encuentran de a pares. Tejido fibroso abundante de tipo fibrotraqueido. Radios uniseriados y perforaciones simples. Puntuaciones escalariformes u opuestas.

De esta manera, la madera de ambos fragmentos fue identificada como coigüe (Nothofagus dombeyi (Mirbel) Oersted) o coigüe de Chiloé (Nothofagus nitida), sin poder lograr hacer la distinción entre estas dos especies a nivel anátomico. Esta madera ha sido descrita como fácil de trabajar, de resistencia mecánica media y de resistencia moderada contra hongos e insectos. Entre sus usos se describe el uso en embarcaciones (Hall \& Witte, 1998).

\section{Fechado $C^{14}$}

Se envió una pequeña muestra de madera del Fragmento 1 para ser analizado mediante el método de $\mathrm{C}^{14}$ en el laboratorio Beta Analytic. El análisis fue realizado mediante el método de AMS, y su resultado se resume en la tabla 3 .

Lamentablemente este análisis no tuvo el resultado, o más bien la precisión esperada y que se necesita para períodos recientes, situando al fragmento 1 en un rango temporal bastante amplio entre los años 1.672 y $1.949 \mathrm{dC}$ años calibrados. Sin embargo, el fechado convencional da un resultado de 160 +/- 40 AP. Se debe considerar que la documentación plantea que las dalcas habrían subsistido solamente hasta finales del siglo XIX en el archipiélago de Chiloé (Finterbusch, 1934; Medina, 1984), por lo que podríamos considerar la fecha de 1900 como la más tardía para el uso de las dalcas en esta zona. Sin embargo, siempre cabe la posibilidad de que se hayan conservado en algunos lugares más remotos y aislados por un mayor intervalo de tiempo.

\section{CONCLUSIONES}

Ambos fragmentos se encuentran altamente erosionados en su superficie, lo que dificulta la observación de huellas de manufactura y de uso en esta primera observación.

Los agujeros de ambos tablones son muy distintos, siendo los del Fragmento 2 más grandes pero con menor diferencia entre cara exterior $e$ interior debido al menor grosor del tablón. También se aprecia desgaste posible por las costuras. Todo esto puede hacer pensar que se trata de fragmentos de dalca de dos tablas distintas, o al menos de dos extremos distintos del mismo tablón, aunque es necesario realizar mayores observaciones para afirmar estas posibilidades. Se sugiere que ambos fragmentos corresponderían a extremidades de tablones de dalca que formaban las bordas de la embarcación (Fig. 1 b), el Fragmento 1 a la parte inferior de un tablón de borda, y el Fragmento 2 a la extremidad superior de otro. Estas inferencias se han realizado tomando en consideración la forma, los ángulos y curvaturas de ambos fragmentos, y a partir de la comparación con otros ejemplares que hemos podido observar y estudiar parcialmente en algunos casos, como los de la dalca del lago Chapo

Tabla 3. Fechado $\mathrm{C}^{14}$ sobre el Fragmento $1^{2}$.

\begin{tabular}{|c|c|c|c|c|}
\hline Muestra & $\begin{array}{c}\text { Código } \\
\text { laboratorio }\end{array}$ & $\begin{array}{c}\text { Fechado C } \\
\text { Convencional }\end{array}$ & Calibración con un sigma & $\begin{array}{c}\text { Calibración con } 2 \text { sigmas (95\% } \\
\text { probabilidad) }\end{array}$ \\
\hline DACH1-F1 & Beta-277775 & $160+/-40 \mathrm{AP}$ & Cal. 1682 a $1949 \mathrm{dC}$. & Cal. 1672 a $1949 \mathrm{dC}$. \\
\hline
\end{tabular}

2 Para la calibración del fechado se utilizó el programa Calib 7.01 y la curva SHCal13. 
y los del Museo de Gotemburgo y Estocolmo en Suecia. Los fragmentos de Achao deben ser entendidos a la luz de estas informaciones, junto con las pocas ilustraciones de dalcas que existen. De esta manera el Fragmento 1 describe una curvatura que correspondería a la parte inferior de una borda (Fig. 1 b), mientras que el Fragmento 2 al presentar agujeros tanto en su parte superior como inferior correspondería a uno de los vértices de la borda (Fig.1 b). No es posible asegurar que ambos fragmentos sean parte de una misma embarcación, (por lo que se hace necesario tomar a cada fragmento como una pieza independiente, y su descripción y de sus agujeros se realiza por separado) aunque probablemente haya sido así. Sin embargo, nuevamente debemos decir que análisis más profundos deben realizarse en este sentido para corroborar esta propuesta.

Hasta el momento no se conoce en detalle la manera en que eran manufacturados los agujeros, ni las herramientas que se empleaban ${ }^{3}$, sin embargo, a partir de lo observado en estas piezas, podemos proponer que probablemente se realizaba un trazado cuadrangular con una herramienta similar a un formón, el cual se realizaba desde la cara interior hacia el exterior en el caso de ambos fragmentos. Este trazado se iba reduciendo en tamaño a medida que se profundizaba en el desbaste de la madera, por lo que es de mayor tamaño por donde se inició que por donde atravesó al otro lado. Esto es similar a lo que se observó en la dalca del lago Chapo, donde los agujeros también son de mayor tamaño por la cara interior del tablón, lo que se explicaría por una necesidad de que la cara de la embarcación que queda expuesta al contacto con el agua presente una menor superficie por donde ésta pueda ingresar y sea más fácil de sellar. Sin embargo, los agujeros de la dalca de lago Chapo presentan una morfología diferente, más bien circular. Por su parte, Bird (1938, p. 254) reporta que el fragmento que observó presentaba agujeros que habrían sido manufacturados con algún tipo de taladro rotador. Ha quedado pendiente para una próxima oportunidad la medición de la distancia entre los agujeros y de éstos con el borde del tablón, dato que sí fue tomado en el caso de la dalca del lago Chapo, y que serviría para fines comparativos.

3 Aunque las fuentes escritas describen a grandes rasgos la manufactura de las dalcas y de los agujeros, se desconoce
A pesar de la erosión superficial, se registraron huellas de exposición al fuego $\mathrm{y}$ carbonización en la superficie del Fragmento 1. Esto coincide con lo detallado por las fuentes escritas para el proceso de manufactura y con las observaciones realizadas en los ejemplares de Chapo y Gotemburgo. En el Fragmento 2, no se observó carbonización de la superficie. Esto puede deberse tanto a la erosión a la que se ha visto expuesto como al hecho de que se trata de un pequeño fragmento, lo que no permite afirmar que el resto del tablón no haya sido expuesto al fuego en su proceso de manufactura.

Como mencionáramos anteriormente, la fecha entregada por el análisis de $\mathrm{C}^{14}$ para el Fragmento 1 es bastante amplia y poco concluyente. Sí nos permite decir que éste probablemente no sería anterior a 1670, y que podria situarse entre esta fecha y finales del siglo XIX con mayores probabilidades según lo expresado por las fuentes escritas. Sin embargo, mayores precisiones pueden lograrse en el futuro con nuevos análisis, particularmente del Fragmento 2.

Junto con esto y en forma complementaria, debiera realizarse en un futuro un análisis arqueo - dendrométrico (Lira et al. 2012; Lavier et al. 2005) más detallado que incluya las huellas y el proceso de manufactura, la forma de trabajar la madera, cómo fue tallado y desbastado cada tablón, e intentar restituirlo a su posición dentro del árbol de origen (morfología de la madera y del árbol). También sería útil estudiar la posibilidad de aplicar análisis de dendrocronología, que permitan conocer nuevos datos respecto de estas piezas y su cronología.

Finalmente, en cuanto a la identificación de la especie/taxa de la madera como coigüe (Nothofagus dombeyi (Mirbel) Oersted) o coigüe de Chiloé (Nothofagus nitida) se condice con la abundancia de esta especie en la región y con la calidad de su madera para la manufactura de embarcaciones. El coigüe ya ha sido reportado para la construcción de canoas monóxilas, especialmente en la zona de Nahuelhuapi (Braikovich, 2004; Lira, 2015). Los fluidos contactos e intercambios con esta zona desde Chiloé, los cuales se encuentran ampliamente documentados a través de las

el detalle del procedimiento para lograr perforar la madera, así como las herramientas utilizadas. 
fuentes escritas -donde se describen los viajes de exploración y misionales en dalcas saliendo desde Chiloé (Fernández, Menéndez, Güell y Fonck) (Hanisch, 1982; Urbina, 2008)- nos hablan de un circuito de navegación y de embarcaciones muy bien adaptadas a estos ambientes. Por otra parte permite seguir cuestionando la tesis de que las dalcas se habrían construido en madera de alerce, idea consolidada a nivel académico (Lira, 2006; Carabias et al. 2007), pero que hasta el momento no tiene su correlato material. Continuando con esta idea es importante referenciar que los fragmentos de dalca del lago Chapo son de madera de ulmo (Eucryphia cordifolia Cav), mientras que podemos adelantar que los análisis preliminares de la dalca de Gotemburgo evidenciaron que se trataría de coigüe. Según Cooper (1917) las fuentes documentales del siglo XVIII y XIX explicitan el uso de Nothofagus. Hasta el momento los análisis que se han realizado al respecto no sustentan la idea del uso del alerce para la construcción de las dalcas, o al menos muestran una mayor variación en la selección de especies que la que se pensaba. Claramente los fragmentos de dalca a los cuales se les ha realizado una identificación anatómica en laboratorio, es decir los fragmentos de dalca de lago Chapo y de Achao, junto con la dalca de Gotemburgo, han dado como resultado especies distintas al alerce. Por esto podemos decir que hasta el momento no se han encontrado evidencias materiales del uso de alerce para la manufactura de dalcas. A modo de síntesis, los fragmentos de dalca de Achao permiten profundizar la discusión acerca de la idea del uso del alerce en la construcción de las dalcas como algo transmitido, sin un mayor fundamento material. Este es un tema que consideramos debiera seguirse discutiendo en el futuro con nuevos hallazgos.

Es importante recordar la importancia material de estos restos de dalca, por la escasez de este tipo de evidencias. De la misma forma, los fragmentos de dalca de Achao son los segundos de su tipo que han sido estudiados en forma sistemática por un equipo de arqueólogos.

\section{AGRADECIMIENTOS}

Los autores quieren agradecer muy especialmente a Ana Rosa Uribe, presidenta de la
Agrupación del Museo Etnográfico de Achao, por todas las facilidades y buena disposición para con nosotros durante este estudio; a Felipe Montiel, por su acogida en el museo de Castro y el contacto con Ana Rosa Uribe; al Dr, Juán Eduardo Diaz-Vaz por su ayuda en la identificación de la especie de la madera; y a la Dra. Leonor Adán, directora de la Dirección Museológica de la Universidad Austral de Chile e investigadora responsable del proyecto Fondecyt 1060216 a partir del cual se financió el fechado de radiocarbón. También a la Dra. Ximena Urbina por sus comentarios y observaciones. Este trabajo se enmarca en estudios de doctorado financiados por CONICYT a través del programa Becas Chile.

\section{BIBLIOGRAFÍA}

Bird, J. (1938). Antiquity and migrations of the early inhabitants of Patagonia. Geographical Review, 28(2), 250-275

Braicovich, R. (2006). Las Canos Monóxilas del lago Nahuel Huapi. En P Furnari y F. Brittez (Eds.), Arqueología Histórica en América latina Temas y Diluciones recientes. (pp. 49-70). Mar del Plata, Ediciones Suárez

Carabias, D., Lira, N. \& Chapanoff, M. (2007) Documentación, análisis e interpretación de los restos de una dalca procedente del lago Chapo, región de Los Lagos. En F. Morello, M. Martinic, A. Prieto \& G. Bahamonde (Eds), Levantando piedras, desenterrando huesos... y develando arcanos, VI Jornadas de Arqueología de Patagonia (pp. 257-267). Punta Arenas, Ediciones CEQUA.

Cooper, J. M. (1917) Anaytical and critical Bibliography of the Tribes of Tierra del Fuego and adjacent territory, Bureau of American Ethnology, Bulletin 63, Washington.

Finterbusch, C. A. (1934). Las Dalcas de Chiloé y los Chilotes, Revista Chilena de Historia y Geografía, Tomo LXXV, 82, 412-433.

Fonck, F., (1900). Viajes de Fray francisco Menéndez a Nahuel Huapi. Valparaíso: Editorial F. Niemeyer.

Hall, M. \& Witte, J. (1998). Maderas del Sur de Chile: árboles, aplicaciones y procesos. Santiago de Chile, Editorial Universitaria.

Hanisch, W. (1982). La isla de Chiloé, capitana de rutas australes. Academia superior de ciencias pedagógicas de Santiago.

Latcham, R. E. (1930). La dalca de Chiloé y los canales patagónicos. Boletín del Museo Nacional de Chile, 
Tomo XIII, 63-72.

Lavier, C., Locatelli, C. \& Pousset, D. (2005). De l'artefact en bois à la nature forestière: quelques histoires parlantes. La Revue Forestière Française, LVI, numéro spécial, "le bois de ses origines à nos jours", 17-29.

Lira, N. (2015). Embarcations de tradition indigéne en Patagonie du Nord/ Sud du Chili: connexions, contacts et routes de la cordillere des Andes à la mer. Tesis de doctorado presentada a la Universidad de Paris 1 Panthéon-Sorbonne.

Lira, N. (2006). MS. Práctica profesional para la Licenciatura de Arqueología, "Registro, análisis y puesta en valor de tres fragmentos de dalca del lago Chapo, X región" profesora guía Victoria Castro. Universidad de Chile.

Lira, N. \& Legoupil, D. (2014). Navegantes del sur y las regiones australes. En Carlos Aldunate (Ed.), Mar de Chile (pp
102-143). Santiago, Ediciones del museo chileno de arte precolombino.

Lira, N., Díaz-Vaz, J. E. \& Muñoz, S. (2012). Canoas monóxilas del centro sur de Chile: estado de conservación y estructura celular de la madera, Magallania, 40(1), 307-318.

Lothrop, S. K. (1932). Aboriginal Navigation Off the West Coast of South America. The journal of the Royal Anthropological Institute of Great Britain and Ireland, 62, 229-256.

Medina, A. (1984). Embarcaciones chilenas precolombinas: La Dalca de Chiloé. Revista Chilena de Antropología, 4, 121-138

Urbina, X. (2008). La frustrada misión estratégica de Nahuel Huapi, un punto en la inmensidad de la Patagonia. Magallania, 36(1), 5-30. 